\title{
Nuclear Cataract Grade 3
}

National Cancer Institute

\section{Source}

National Cancer Institute. Nuclear Cataract Grade 3. NCI Thesaurus. Code C135195.

A stage of nuclear sclerotic cataract marked by pronounced yellowing and sclerosis of the lens nucleus. (Modified LOCS II) 